\title{
Postoperative Craniotomy Pain Management in Pediatric Patients: A Systematic Review
}

\author{
Marie Christelle S Endencia, MD*, Mary Gold C Piñera, MD and Karl Matthew C Sy Su, MD, \\ FPSA
}

Department of Anesthesiology, Philippine General Hospital, University of the Philippines, Philippines

\begin{abstract}
Background: There is increasing evidence supporting increased pain intensity following neurosurgical procedures. There are different approaches to analgesia following craniotomy and cranioplasty, but there is limited consensus on postcraniotomy pain management especially in the pediatric population.
\end{abstract}

Methods: A comprehensive online search was performed using the Preferred Reporting Items for Systematic Reviews and Meta-analyses (PRISMA) guidelines. Literature was taken from PubMed, EMBASE, Science Direct, ProQuest, and Google Scholar databases. Human comparative studies including randomized controlled trials and cohort studies evaluating pain scores after neurosurgery in pediatric patients were included in the review.

Results: A total of 3 RCTs and 6 cohort studies met the inclusion criteria. The heterogeneity of the studies included did not allow for data pooling and statistical analysis. All studies evaluated the efficacy of pharmacologic interventions in pediatric patients who underwent craniotomy by measuring postoperative pain scores. Continuous opioid infusions postoperatively provided favorable postoperative pain control in pediatric patients without serious opioid-induced adverse complications. Intraoperative doses of opioids for preemptive analgesia had favorable outcomes but still lack evidence. Non-opioid analgesics are suitable adjuncts to postoperative opioids to enhance analgesia and minimize adverse events use of local anesthetics as local scalp infiltration or nerve block for children resulted in lower postoperative pain scores and longer time to first rescue analgesia compared to placebo, but still need further studies.

Conclusion: Opioids remain as mainstay treatment for children who underwent neurosurgery but specific recommendation on the method and timing of delivery of opioids cannot be drawn from this review. The use of non-opioid analgesics and local anesthetics for local infiltration and nerve block need further research. There is a lack of high-quality evidence on this field, and additional research is necessary to improve pain management after craniotomy in the pediatric population.

\section{Keywords}

Craniotomy, Pediatric neurosurgery, Pain management, Pediatrics, Pediatric anesthesia

\section{Introduction}

Craniotomies in the pediatric population is a common surgical procedure owing to the high incidence of central nervous system (CNS) tumors in children [1] and an increase in the cases of pediatric traumatic brain injuries [2] requiring surgical management. Despite extensive surgical manipulation, craniotomies were assumed to have mild to moderate postoperative pain [3], therefore, pain assessment on post-craniotomy patients has been subjected to limited research especially in pediatric patients [4]. However, several recent studies suggest that moderate to severe pain in the first 24 to 48 hours postoperatively may be higher than previously expected [5], and postoperative pain following intracranial surgery is more significant than initially reported, with as many as $87 \%$ of patients reporting moderate to severe pain in the first 24 hours post-procedure $[6,7]$.
Post-craniotomy pain is predominantly superficial, suggesting somatic origin [8] originating from the scalp, muscles, and soft tissue, with subsequent activation of the pain pathway from manipulation of the dura mater [9]. Post-craniotomy pain is usually localized to the surgical site and surrounding structures and results from incision and traction during surgery [10]. The nature of post-craniotomy pain is described

*Corresponding author: Marie Christelle S Endencia, MD, Department of Anesthesiology, University of the Philippines, Philippine General Hospital, Philippines

Accepted: March 13, 2021

Published online: March 15, 2021

Citation: Endencia MCS, Piñera MGC, Sy Su KMC (2021) Postoperative Craniotomy Pain Management in Pediatric Patients: A Systematic Review. J Clin Anesth Pain Manag 5(1):205-213 
as pounding or pulsating pain [8], and persistent pain observed in some patients is proposed to be from dural irritation, pericranial muscle retraction, decreased cerebrospinal fluid pressure, and aseptic meningitis [11], with tension headache and neck muscle spasm from surgical positioning contributing to postoperative pain [9]. Several studies have cited sex, age, and preoperative opioid use as correlative to increased post-craniotomy pain [11], while other studies show that craniotomy site influences postoperative pain, showing a trend of higher pain scores in patients who underwent posterior fossa and temporal craniotomies due to increased muscle mass incised and retracted during surgery [12]. Studies have also demonstrated that the choice of anesthetic used intraoperatively may be a factor in post-craniotomy pain, with inhalational anesthesia associated with higher postoperative pain [9].

There is still no consensus on the approach to postoperative craniotomy pain. The uses of local anesthetics, NSAIDs, and opioids have been studied in adult patients for pain control after craniotomy. Regional anesthesia is employed through (1) Scalp block, used as preemptive analgesia, therefore decreasing the need for rescue pain medications at the Post-Anesthesia Care Unit [13], and (2) Local anesthetic infiltration on surgical incision, which produces a modest decrease in postsurgical pain scores up to an hour following surgery $[14,15]$. Nonopioid analgesics, including COX inhibitors and acetaminophen offer opioid-sparing effects, but may be inadequate if used alone post-neurosurgery [9]. Acetaminophen is ubiquitously used in pediatric neurosurgical practice due to its relatively high safety profile, with anti-pyretic and minimal anti-inflammatory activity [5]. COX-inhibitors are also used to manage post-craniotomy pain, with caution for possible risk of postoperative hematoma [16] and cardiovascular thromboembolic disease [16]. Parenteral opioids provide superior analgesia in patients who underwent major surgery. Opioids remain a mainstay in the management of post-craniotomy pain in the adult and pediatric population $[17,18]$. The more commonly used parenteral opioids are morphine and fentanyl in intermittent boluses or PCA infusion which reduce pain scores in post-craniotomy patients especially when used in conjunction with NSAIDs [5].

Despite the number of modalities available in managing post-craniotomy pain, neurosurgical patients have been subjected to undertreatment of postoperative pain due to a presumed lack of need for a more aggressive approach to pain control [19]. There is apprehension in the administration of opioids in the postoperative period, given concerns of miosis and sedation from overzealous pain management that can prevent the performance of accurate neurologic examinations and possibly conceal signs of intracranial emergencies [8]. In addition, the undesired respiratory depression from opioids can cause hypercapnia and cerebral vasodilation that may lead to neurosurgical complications, among them cerebral edema and increased intracranial pressure [20]. The minimal use of opioids based on issues regarding opioid-induced complications is further reinforced by the belief that neurosurgical procedures have modest pain $[21,22]$, ultimately causing failure to adequately treat pain in the postoperative period. However, it is prudent to address post-craniotomy pain because poorly controlled craniotomy pain in negatively impact hemostasis and cerebral hemodynamics $[6,7]$ causing cerebral hemorrhage and development of chronic pain [23]. Therefore, aggressive management of pain should be the prevailing standard of care [24].

Considering the limited literature on this matter, this systematic review aims to consolidate all available literature related to pharmacologic modalities for the pain control after craniotomy in the pediatric population. Specifically, this review will focus on the postoperative pain scores and rescue analgesic dose requirements after pharmacologic intervention in a pediatric patient for craniotomy or cranioplastic surgery.

\section{Methods}

A systematic review was done according to the Preferred Reporting Items for Systematic Reviews and Meta-analyses (PRISMA) without a formal registration number. A comprehensive literature search was conducted to identify relevant comparative studies on postoperative craniotomy pain management modalities on pediatric patients indexed in PubMed, EMBASE, Science Direct, Cochrane Library, ProQuest, and Google Scholar. The search terms ("craniotomy or neurosurgery"), "pain", and ("pediatric or children") were used with no date or language restrictions. The final search in all databases was performed on October 10, 2020.

Two initial reviewers (M.C.S.E. and M.G.C.P.) screened and assessed the titles and abstracts before full manuscripts were retrieved, then reviewed for eligibility according to the set inclusion criteria. All disagreements concerning study selection and inclusion were resolved through consensus with a third reviewer (K.M.C.S.).

The inclusion criteria for this review were human comparative studies including randomized controlled trials (RCT), and both retrospective and prospective cohort studies on modalities of pain management post-craniotomy in pediatric patients. Literature that assessed post-craniotomy pain in children using numeric or behavioral pain scales or additional analgesic consumption were considered. All studies that included manipulation of the cranium including emergency and elective craniotomies for tumor excision, cranioplasty, or cranial reconstructive surgeries, and cranial vascular repair were considered for review. Studies included in the final review may or may not measure secondary outcomes such as adverse effects of the treatment modality being studied.

Case reports, case series, book chapters, conference abstracts, review articles, and articles not written in the English language or unavailability of English-translated full text were excluded from the review. The references from the included studies were retrieved and manually cross-referenced to include any relevant literature that may have been missed during the initial electronic database search.

Data from the manuscripts were extracted and recorded in a dedicated data extraction form. The extracted data included publication details (author, year of publication, and study location), study details (type of surgery, sample size with mean age), interventions (intraoperative analgesia, 


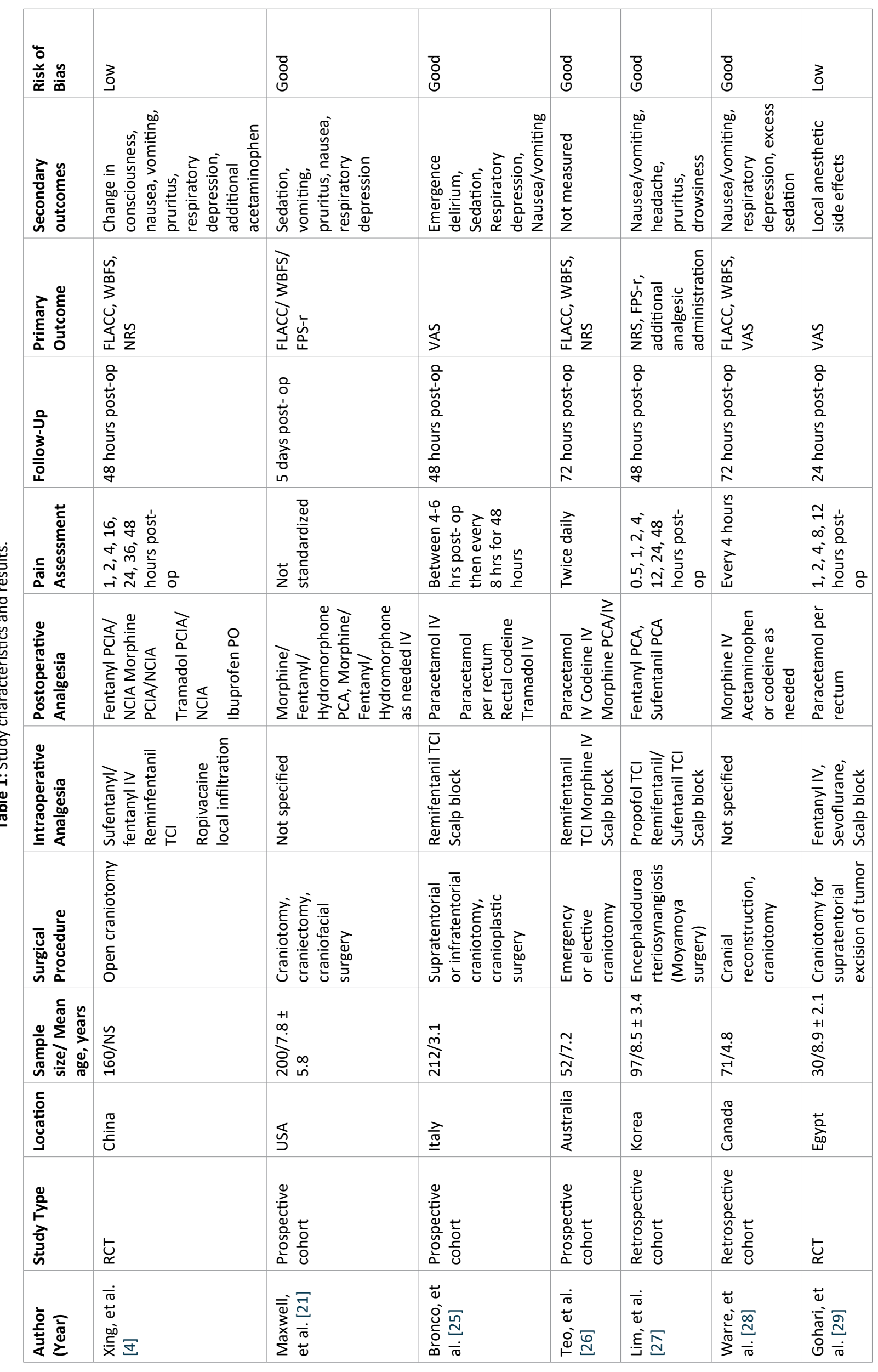




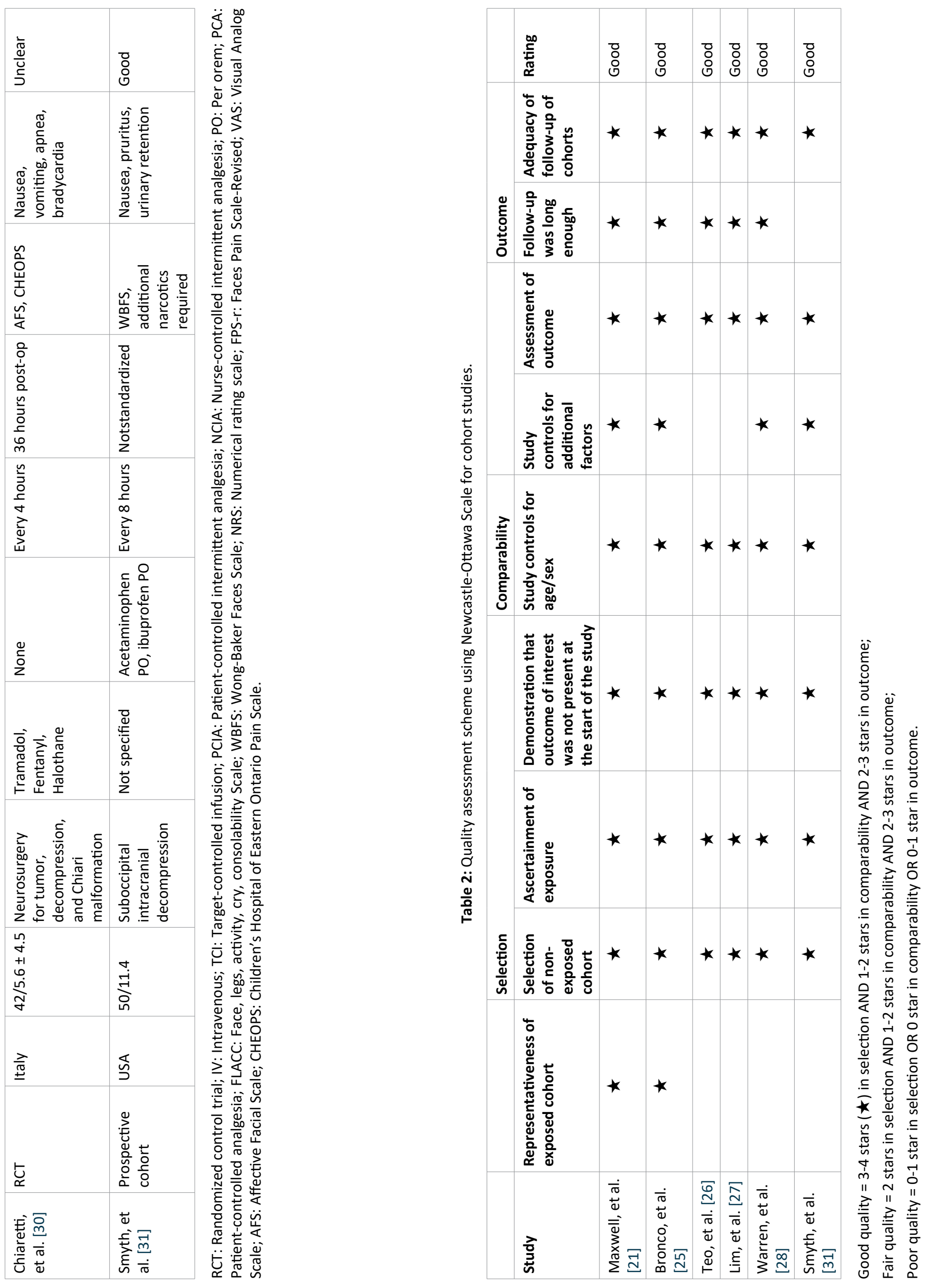


postoperative analgesic modality, drug dosage), and findings of the study. The primary outcome was the efficacy of the studied pain management modality assessed by the pain scores and supplementary analgesia consumption and secondary outcomes were adverse effects secondary to the pain treatment (Table 1) [4,21,25-31].

Assessment of data quality and risk of bias was conducted independently by two authors (M.C.S.E. and M.G.C.P.) using separate scales for the RCTs and non-randomized studies. Risk of bias for the cohort studies was assessed using the Newcastle-Ottawa Scale [32] which considered three factors to score the quality of the study: (1) Selection of the exposed and non-exposed cohorts; (2) Comparability based on the study design and analysis; and (3) Outcome, including cohort retention, follow-up, and blind assessment. Interpretation of scores adapted from the validity and inter-rater reliability testing report of the Agency for Healthcare Research and Quality (AHRQ) were as follows: A "good" quality score required 3-4 stars in selection, 1-2 stars in comparability, and 2-3 stars in outcomes; a "fair" quality score required 2 stars in selection, 1-2 stars in comparability, and 2-3 stars in outcomes; and a "poor" quality score have $0-1$ star in selection, 0 stars in comparability, and 0-1 star in outcomes [33] (Table 2). The risk of bias in the RCTs was assessed using the Cochrane Collaboration Risk of Bias Tool, where domains of selection bias, reporting bias, performance bias, detection bias, and attrition bias were classified as low, high, or unclear [34].

The heterogeneity of the studies and diversity of outcomes included in this review did not allow for data pooling and statistical analysis, therefore results are summarized narratively.

\section{Results}

The search strategy described yielded a total of 9 articles for review, as summarized by the PRISMA flow diagram (Figure 1). Three RCTs and 6 cohort studies (4 prospective, 2 retrospective) met the inclusion criteria and retrieved for fulltext review and included in the final analysis.

\section{Study characteristics}

Characteristics and data were summarized (Table 1) and assessed for validity (Table 2 and Table 3). Postoperative pain after craniotomy was the primary outcome measured
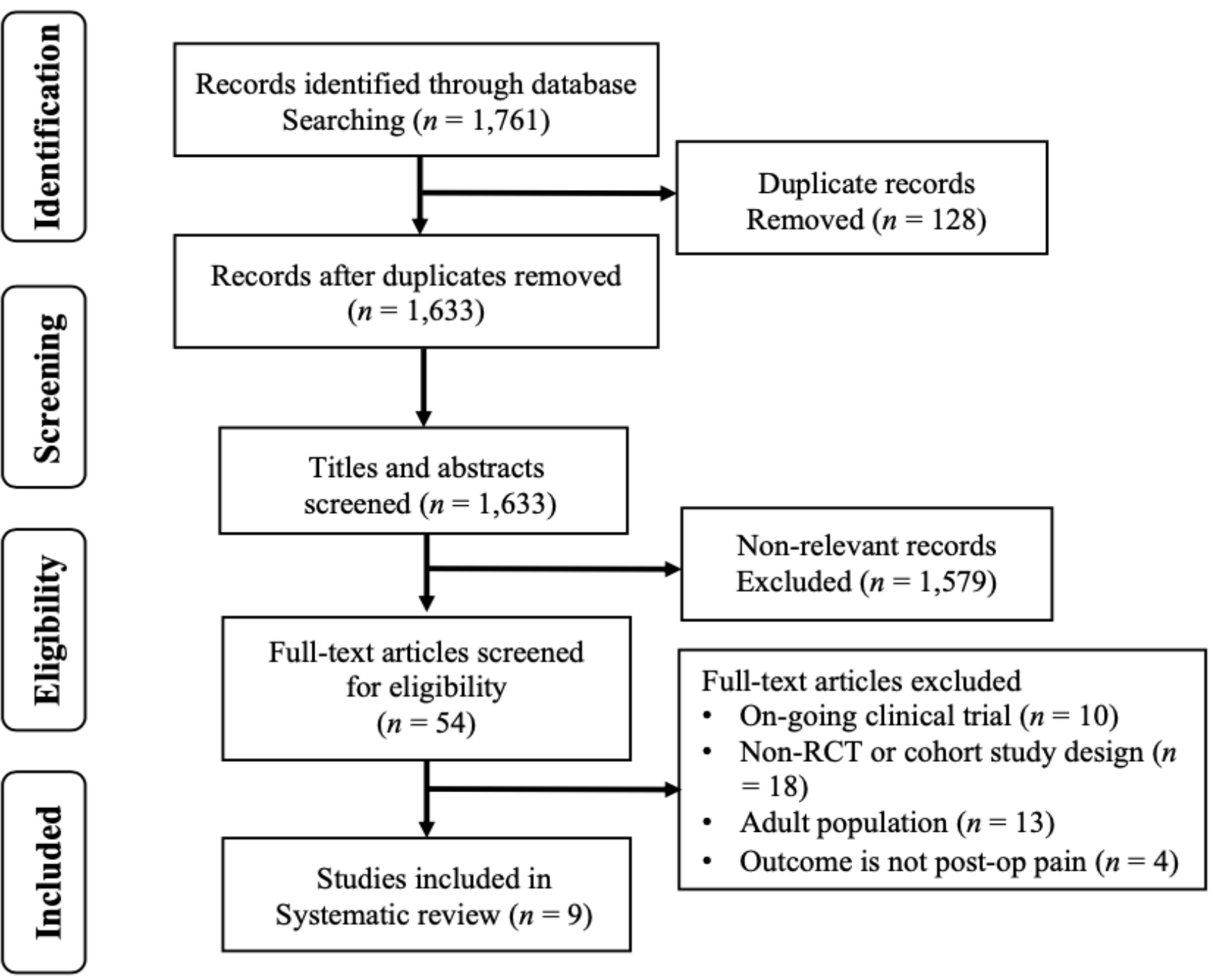

Figure 1: PRISMA flow diagram. 
Citation: Endencia MCS, Piñera MGC, Sy Su KMC (2021) Postoperative Craniotomy Pain Management in Pediatric Patients: A Systematic Review. J Clin Anesth Pain Manag 5(1):205-213

Table 3: Risk of bias assessment using cochrane collaboration risk of bias tool.

\begin{tabular}{|l|l|l|l|l|l|l|}
\hline Study & $\begin{array}{l}\text { Random } \\
\text { sequence } \\
\text { generation } \\
\text { (Selection } \\
\text { Bias) }\end{array}$ & $\begin{array}{l}\text { Allocation } \\
\text { concealment } \\
\text { (Selection Bias) }\end{array}$ & $\begin{array}{l}\text { Selective } \\
\text { reporting } \\
\text { (Reporting } \\
\text { Bias) }\end{array}$ & $\begin{array}{l}\text { Blinding } \\
\text { participants } \\
\text { and personnel } \\
\text { (Performance } \\
\text { Bias) }\end{array}$ & $\begin{array}{l}\text { Blinding } \\
\text { outcome } \\
\text { assessment } \\
\text { (Detection } \\
\text { Bias) }\end{array}$ & $\begin{array}{l}\text { Incomplete } \\
\text { outcome data } \\
\text { (Attrition Bias) }\end{array}$ \\
\hline Xing, et al. [4] & $(+)$ & $(+)$ & (+) & (+) & (?) & $(+)$ \\
\hline Gohari, et al. [29] & (?) & (+) & (+) & (+) & (?) & (?) \\
\hline Chiaretti, et al. [30] & $(+)$ & (?) & (?) & (?) & (?) & (?) \\
\hline
\end{tabular}

$(+)=$ Low risk of bias; (?) = Unclear risk of bias; $(-)=$ High risk of bias.

in all studies, assessed by standardized numeric and behavioral scales, using the Face, legs, arms, cry, consolability (FLACC) Scale $[4,21,26,28]$, Wong-Baker Faces Scale (WBFS) [4,21,26,28,31], Faces Pain Scale-Revised (NPS-r) [21,27], Numerical Rating Scale (NRS) $[4,26,27]$, Visual Analog Scale (VAS) $[25,28,29]$, Affective Facial Scale (AFS) [30], Children's Hospital of Eastern Ontario Pain Scale (CHEOPS) [30], or a combination of these scales depending on the age of the patients being assessed, and additional required analgesic postoperatively $[4,27,29]$. Secondary outcomes were measured in 7 out of the 9 studies reviewed and the adverse effects of opioids $[4,21,25,27,28,30,31]$, one study assessed the adverse effects of local anesthetics [29], and one study did not measure any secondary outcome. Only perioperative pharmacologic treatments either through systemic drug treatment or use of local anesthetics as local scalp infiltration of scalp nerve block were employed in all the studies reviewed.

One study had a 24-hour post-craniotomy follow-up period [29], one study had a 36-hour follow-up [30], 3 studies reported results up to 48 hours postoperatively $[4,25,27]$, while 3 studies extended the follow-up to 72 hours $[26,28]$ and 5 days post-craniotomy [21]. One study recorded the pain scores until they were discharged with no fixed follow-up time [30].

\section{Summary of findings}

Systemic pharmacologic treatment: An RCT compared the incidence of moderate-to-severe post-craniotomy pain scores and total dose of rescue medications in three different opioid patient-controlled analgesia (PCA) protocols (fentanyl vs. morphine vs. tramadol) compared to placebo in younger (1-6 years-old) and older (7-12 years-old) children age groups. It showed that patients in both the younger and older age groups receiving morphine PCA had statistically significant lower pain scores than those receiving fentanyl, tramadol, and placebo, with no significant difference in pain scores between tramadol and fentanyl PCA group [4]. Moreover, oral ibuprofen or morphine rescue doses were significantly higher in the control groups compared to the groups receiving opioid PCA infusions, with no significant difference between opioid-induced complications across all treatment groups and the placebo [4]. Similarly, a trend towards better pain control was seen in children receiving continuous morphine infusions after cranial surgery compared to those who did not receive morphine infusions postoperatively. [28]. In addition, children who received parenteral morphine given intraopera- tively as a loading dose followed by postoperative parenteral morphine delivered either as an infusion or through PCA generally had lower pain scores over the 72 postoperative hours [26]. However, it was seen that there is a higher incidence of nausea in patients receiving continuous morphine infusion on their first post-op day [28].

Postoperative pain scores in children after Moyamoya surgery who received fentanyl and sufentanil through PCA were measured using median pain scores and additional rescue doses of ketorolac postoperatively. It showed equally effective post-operative pain control in both fentanyl and sufentanil PCA groups, however, the use of additional ketorolac was significantly higher in patients receiving fentanyl PCA [27]. In the same manner, a prospective observational study supports that craniotomy and cranial reconstruction pain are common among pediatric patients but effectively controlled using hydromorphone, fentanyl, or morphine administered intravenously either by PCA or by nurses on an as-needed basis, with no significant difference in pain scores between the different delivery groups [21]. The total opioid consumption of patients using opioid PCA was 3-5 times higher than those with intermittent as-needed administration [21], resulting in an increase in incidence of nausea and vomiting but was not associated with respiratory depression or neurologic deterioration $[4,21]$.

The value of opioids in preemptive analgesia has been shown in an RCT wherein an intraoperative bolus or infusion of tramadol or fentanyl was delivered to control post-neurosurgery pain in children [30]. Even without a scheduled postoperative analgesic regimen, children who received tramadol bolus, tramadol bolus, and infusion, and fentanyl infusion still had low postoperative pain scores at PICU where only 2 out of 42 participants required additional analgesia postoperatively [30].

The analgesic efficacy and safety of multimodal systemic pharmacologic analgesia using opioids with NSAIDs and paracetamol were assessed in several cohort studies. Paracetamol and ibuprofen were the common choices of non-opioid analgesics in children after craniotomy. Acetaminophen (paracetamol) and ibuprofen given postoperatively reduced postoperative pain scores and rescue narcotic requirements in children who underwent suboccipital decompression, especially when administered as a scheduled regimen [31]. These non-narcotics, in combination with rectal codeine or intravenous tramadol, afforded excellent post-craniotomy 
pain relief in children with a median pain score of 1 , and only $16-19 \%$ of children presenting with moderate to severe pain in PACU up to the second post-op day [25]. Multimodal analgesia minimizes opioid-related side effects as demonstrated by the common use of acetaminophen alone or in combination with opioids in children after neurosurgery, wherein 75 $92 \%$ of patients received acetaminophen on their first postoperative day $[21,26]$.

Local scalp infiltration or nerve block: Randomized trials and cohort studies assessed the use of local anesthesia as local scalp infiltration or scalp nerve block in pediatric patients for cranial surgery. The application of scalp nerve block using bupivacaine $0.25 \%$ before surgical incision in children for elective supratentorial craniotomy significantly decreased intraoperative fentanyl requirements and prolonged the time to first analgesic rescue dose in the first 24 hours after surgery [29]. Children who received scalp nerve block also had significantly lower VAS scores in the first 12 hours postoperatively compared to placebo, with no reported incidence of local or systemic side effects from local anesthesia [29].

Multimodal analgesia employing the use of scalp infiltration using bupivacaine and epinephrine combined with postoperative opioids also produced lower median pain scores in children [26]. However, the timing of local infiltration may affect the postoperative pain scores, with children receiving local anesthesia before and/or after surgery presenting with lower median pain scores compared to those who received local anesthesia only prior to incision [25].

\section{Discussion}

Pain management in the pediatric population poses a challenge due to their inability to describe or accurately express their pain. Some children are also hesitant in voluntarily verbalizing discomfort for fear of further interventions [28]. While increasing evidence has shown that pain following neurosurgery in adults is more significant $[6,7,35]$ than previously estimated, there is still limited research on post-craniotomy pain management in the pediatric population. Even in the systematic review of postoperative pain in pediatric patients from Ross, et al. [36], pain following neurosurgical procedures in children was not assessed. This systematic review aims to summarize the efficacy and safety of the pain management modalities employed in children. There is a paucity of randomized controlled trials involving pediatric patients, therefore the interventions assessed in this review are variable.

Inadequate pain control following neurosurgery can have consequences leading to significant morbidity including systemic and intracranial hypertension, intracerebral hematoma, and seizures $[6,7,23,37]$. The studies reviewed assessed pharmacologic interventions employed in children for neurosurgery and varied in terms of methodology, assessed outcomes, interventions, and length of the follow-up period.

Although there have been previous concerns regarding the use of opioids following neurosurgery due to its undesired sedative and respiratory effects [8], parenteral opioids remain as the gold standard in managing moderate to severe pain and has gained popularity even in the pediatric population.
Preemptive analgesia in the form of intraoperative opioid infusion is rarely considered in the pediatric population but has shown promising results in reducing postoperative pain scores after craniotomy [30]. This is in contrast to a previous meta-analysis concluding that preemptive systemic opioid administration is not effective in reducing postoperative pain scores [38]. Nevertheless, the use of opioids as postoperative infusions consistently produces favorable results in providing safe and improved pain control in children $[4,21,28,30]$ due to better clearance and blood distribution [22]. Although the incidence of nausea and vomiting may be increased in children who received continuous opioid infusions [4,28], more serious opioid-related complications such as respiratory depression and altered neurologic status were not evident in any of the studies $[4,21,25-28]$ included. Furthermore, the incidence of nausea and vomiting persisted despite discontinuation of morphine infusions [28] or concomitant naloxone infusion [21], suggesting that other etiologies of postoperative vomiting may have been present in these patients [28]. In addition, combining them with non-opioid analgesics such as paracetamol and NSAIDs as part of a multimodal analgesic regimen not only improves their safety profile but establishes enhanced analgesic value $[25,26]$.

There are varying protocols on the delivery of pain management per institution. Opioid delivery as PRN (pro re nata or as-needed) dose is prompted only when the patient expresses pain [21]. However, several factors limit the efficacy of this analgesic delivery especially in the younger pediatric population. This includes the inherent delay between the occurrence of pain and administration of opioids [39], the inability to verbalize increased pain in younger children, and inadequate dosing and timing intervals from the prescriber's lack of familiarity or fear of opioid-induced side effects [21]. Moreover, PRN administration of opioids may result in alternate periods of inadequate analgesia and excessive sedation [35]. Findings from Maxwell, et al. [21] showed that the use of PCA with background infusion using hydromorphone, fentanyl, or morphine in pediatric patients after craniotomy is safe and effective in reducing pain scores similar to the adult population receiving fentanyl [39] or morphine [40] based PCA treatment.

Nonsteroidal anti-inflammatory drugs offer satisfactory analgesic effects [8] for acute post-operative pain, and are a safe adjunct for pain control in children undergoing craniotomy [41]. Children who received scheduled doses of acetaminophen and ibuprofen after cranial decompression surgery experienced lower postoperative pain scores and required fewer rescue doses of narcotics and anti-emetics [31]. Despite previous restrictions on the use of NSAIDs due to their antiplatelet mechanism [39], non-opioid analgesics including COXIBs and acetaminophen are still commonly employed in pain management of children undergoing neurosurgery $[21,25,26]$ to produce little to no pain at PACU $[25,26]$. However, it should be noted that the timing of delivery of these medications matters significantly, and regular, scheduled administration of acetaminophen or ibuprofen delivers more benefit than PRN doses [31].

Multimodal strategy for pain control in neurosurgery 
includes the use of local anesthesia for scalp infiltration or nerve block [8]. Randomized trials on the use of local anesthesia for pain management in adults have shown that regional scalp block improves recovery profiles of patients after neurosurgery by decreasing pain scores and reducing the postoperative need for opioids [42-44]. Likewise, cohort studies on the use of local anesthetics for local scalp infiltration or scalp block in children produced similar results of lower VAS scores and longer time to first rescue analgesia [29]. Yet, it is important to consider that local infiltration and peripheral nerve block only offer a short-term advantage in reducing pain scores after major craniotomy, and factors such as the timing of infiltration and duration of surgery should be considered [10]. Evidence on the use of local anesthesia in the pediatric neurosurgical patient is very limited and randomized trials are yet to be performed. Nevertheless, the safety profile of this technique even in children has been demonstrated [29] and adult findings are promising, therefore it should still be considered as an acceptable adjunct to systemic analgesia after pediatric cranial surgery.

\section{Limitations}

This review is limited by the paucity of available literature on pediatric pain management especially on the neurosurgical population. This study included all accessible comparative studies on pediatric pain and neurosurgery including randomized trials and cohort studies, yielding heterogeneous methods and interventions. Although there were no language restrictions applied during the search, studies with no English-translated full text were eventually excluded, further limiting records. However, as more randomized studies are conducted, standardization of outcome assessment and data collection intervals will allow for data pooling, comparison, and generalizability of outcome.

\section{Conclusion}

This systematic review evaluated studies on pain management post-craniotomy in pediatric patients. This review cannot provide specific recommendations on systemic pharmacologic treatment due to significant variability in methodology and standardization of data collection of the studies included in the review. Opioid treatment to control post-craniotomy pain in children is commonly employed but the method and timing of delivery still varies across different institutions. Non-opioid analgesics and local anesthetics are also used after craniotomy procedures in pediatrics but further studies are needed. In general, multimodal approach is still the best way to manage pain after craniotomy in the pediatric population.

There exists a gap between the use of evidence-based medicine and the availability of research specific for pain management in children undergoing neurosurgery. Non-pharmacologic pain management strategies used in adults could be explored for its applicability in pediatric patients. Finally, further research that will identify specific components of safe multimodal analgesia following neurosurgery and the most optimal method of pain and safety monitoring will be beneficial in the pediatric population.

\section{Funding}

All costs necessary for the implementation of this study was shouldered by the primary author.

\section{References}

1. Silva AHD, Aquilina K (2020) Surgical approaches in pediatric neuro-oncology. Cancer Metastasis Rev 38: 723-747.

2. Leetch AN, Wilson B (2018) Pediatric major head injury: Not a minor problem. Emerg Med Clin North Am 36: 459-472.

3. Nair S, Vedantam R (2010) Evaluation of pain following supratentorial craniotomy. Br J Neurosurg 25: 100-103.

4. Xing F, Li XA, Xue FS, et al. (2019) Postoperative analgesia for pediatric craniotomy patients: A randomized controlled trial. BMC Anesthesiology.

5. Gottschalk A, Yaster M (2007) Pain management after craniotomy. Neurosurgery Quarterly 17: 64-73.

6. Woodall MN, Todnem ND, Withrow J, et al. (2017) Peri-operative pain control in the neurosurgical patient. J Clin Exp Neuroimmunol 2: 1-5.

7. Hansen MS, Brennum J, Moltke FB, et al. (2013) Suboptimal pain treatment after craniotomy. Dan Med J 60: A4569.

8. Vadivelu N, Kai AM, Tran D, et al. (2016) Options for perioperative pain management in neurosurgery. J Pain Res 9: 37-47.

9. Chowdhury T, Garg R, Sheshadri V, et al. (2017) Perioperative factors contributing the post-craniotomy pain: A synthesis of concepts. Front Med (Lausanne) 4: 23.

10. Guilfoyle MR, Helmy A, Duane D, et al. (2013) Regional scalp block for postcraniotomy analgesia: A systematic review and meta-analysis. Anesth Analg 116: 1093-1102.

11. Flexman A, Ng JL, Gelb A (2010) Acute and chronic pain following craniotomy. Curr Opin Anaesthesiol 23: 551-557.

12. Thibault M, Girard F, Chouinard P, et al. (2007) Craniotomy site influences postoperative pain following neurosurgical procedures: A retrospective study. Can J Anaesth 54: 544-548.

13. Saha P, Chattopadhyay S, Rudra A, et al. (2013) Pain after craniotomy: A time for reappraisal? Indian Journal of Pain 27: 7-11.

14. Batoz H, Verdonck O, Pellerin C, et al. (2009) The analgesic properties of scalp infiltrations with ropivacaine after intracranial tumoral resection. Anesth Analg 109: 240-244.

15. Bloomfield EL, Schubert A, Secic M, et al. (1998) The influence of scalp infiltration with bupivacaine on hemodynamics and postoperative pain in adult patients undergoing craniotomy. Anesth Analg 87: 579-582.

16. Palmer J, Sparrow O, lannotti F (1994) Postoperative hematoma: A 5-year survey and identification of avoidable risk factors. Neurosurgery 35: 1061-1065.

17. Nussmeier N, Whelton A, Brown M, et al. (2005) Complications of the COX-2 inhibitors parecoxib and valdecoxib after cardiac surgery. N Engl J Med 352: 1081-1091.

18. Haldar R, Kaushal A, Gupta D, et al. (2019) Pain following craniotomy: Reassessment of the available options. Biomed Res Int 2015: 509164.

19. Gottschalk A, Yaster M (2008) The perioperative management of pain from intracranial surgery. Neurocrit Care 10: 387-402. 
20. Cold GE, Felding M (1993) Even small doses of morphine might provoke "luxury perfusion" in the postoperative period after craniotomy. Neurosurgery 32: 327.

21. Maxwell L, Buckley G, Kudchadkar S, et al. (2014) Pain management following major intracranial surgery in pediatric patients: $A$ prospective cohort study in three academic children's hospitals. Pediatr Anesth 24: 1132-1140.

22. Lutman B, Bloom J, Nussenblatt B, et al. (2018) A contemporary perspective on the management of post-craniotomy headache and pain. Curr Pain Headache Rep 22: 69.

23. Gray LCD, Matta B (2005) Acute and chronic pain following craniotomy: A review. Anaesthesia 60: 693-704.

24. Basali A, Mascha E, Kalfas I, et al. (2000) Relation between perioperative hypertension and intracranial hemorrhage after craniotomy. Anesthesiology 93: 48-54.

25. Bronco A, Pietrini D, Lamperti $m$, et al. (2014) Incidence of pain after craniotomy in children. Paediatr Anaesth 24: 781-787.

26. Teo JH, Palmer GM, Davidson AJ (2011) Post-craniotomy pain in a paediatric population. Anaesth Intensive Care 39: 89-94.

27. Lim L, Jang YE, Kim EH, et al. (2020) Comparison of the effects of sufentanil and fentanyl in intravenous patient-controlled analgesia after pediatric moyamoya surgery: A retrospective study. Pediatr Neurosurg 55: 36-41.

28. Warren DT, Tim BR, Ou C, et al. (2010) Safety and efficacy of continuous morphine infusions following pediatric cranial surgery in a surgical ward setting. Childs Nerv Syst 26: 1535-1541.

29. Gohary M, Gamil M, Girgis K, et al. (2009) Scalp nerve block in children undergoing a supratentorial craniotomy; $A$ randomized controlled study. Asian Journal of Scientific Research 2: 105-112.

30. Chiaretti A, Viola L, Pietrini D, et al. (2000) Preemptive analgesia with tramadol and fentanyl in pediatric neurosurgery. Childs Nerv Syst 16: 93-99.

31. Smyth MD, Banks JT, Tubbs RS, et al. (2004) Efficacy of scheduled nonnarcotic analgesic medications in children after suboccipital craniectomy. J Neurosurg 100: 183-186.

32. Wells G, Shea B, O'connell D, et al. (2000) The Newcastle-Ottawa Scale (Nos) for assessing the quality of nonrandomised studies in meta-analyses. The Ottawa Hospital Research Institute, Ottawa, ON, Canada.
33. Hartling L, Hamm M, Milne A, et al. (2012) Validity and inter-rater reliability testing of quality assessment instruments.

34. Higgins JPT, Altman DG, Gotzsche PC, et al. (2011) The cochrane collaboration's tool for assessing risk of bias in randomised trials. BMJ 343: d5928.

35. Logan SW, Bilotta F (2017) Postoperative pain control following craniotomy: A systematic review of recent clinical literature. Pain Pract 17: 968-981.

36. Ross A, Young J, Hedin R, et al. (2018) A systematic review of outcomes in postoperative pain studies in paediatric and adolescent patients: Towards development of a core outcome set. Anaesthesia 73: 375-383.

37. Xiong W, Li L, Bao D, et al. (2020) Postoperative analgesia of scalp nerve block with ropivacaine in pediatric craniotomy patients: A protocol for a prospective, randomized, placebo-controlled, double-blinded trial. Trials 21: 1-9.

38. Ong CKS, Lirk P, Seymour RA, et al. (2005) The efficacy of preemptive analgesia for acute postoperative pain management: $A$ meta-analysis. Anesth Analg 100: 757-773.

39. Morad A, Winters B, Stevens R, et al. (2012) The efficacy of intravenous patient-controlled analgesia after intracranial surgery of the posterior fossa: A prospective, randomized controlled trial. Anesth Analg 114: 416-423.

40. Dilmen OK, Akcil EF, Tunali Y, et al. (2016) Postoperative analgesia for supratentorial craniotomy. Clin Neurol Neurosurg 146: 90-95.

41. Nesvick CL, Oushy S, Daniels DJ, et al. (2020) Safety of immediate use of nonsteroidal antiinflammatory drugs after pediatric craniotomy for tumor. J Neurosurg Pediatr 26: 327-333.

42. Hwang JY, Bang JS, Oh CW, et al. (2015) Effect of scalp blocks with levobupivacaine on recovery profiles after craniotomy for aneurysm clipping: A randomized, double-blind, and controlled study. World Neurosurg 83: 108-113.

43. Tuchinda L, Somboonviboon W, Supbornsug K, et al. (2010) Bupivacaine scalp nerve block: Hemodynamic response during craniotomy, intraoperative and post-operative analgesia. Asian Biomedicine 4: 243-251.

44. Bala I, Gupta B, Bhardwaj N, et al. (2006) Effect of scalp block on postoperative pain relief in craniotomy patients. Anaesth Intensive Care 34: 224-227.

DOI: $10.36959 / 377 / 355$

Copyright: (C) 2021 Endencia MCS, et al. This is an open-access article distributed under the terms of the Creative Commons Attribution License, which permits unrestricted use, distribution, and reproduction in any medium, provided the original author and source are credited. 